\title{
Uncertainty and Sensitivity Analysis for a Model of an Electric Submersible Pump Lifted Oil Field
}

\author{
Roshan Sharma and Bjørn Glemmestad
}

\begin{abstract}
Dynamic and mechanistic models of an Electrical Submersible Pump (ESP) lifted oil field are frequently used for understanding the process dynamics and the interactions occurring between the oil wells under varying operating conditions. They are also used to calculate the total fluid produced from the oil field. In this article, uncertainty and sensitivity analysis of such a model is studied. If the model is used for control and optimization of an oil field, it is important to see if the output calculated using the mathematical model lies within a confidence interval under the presence of uncertainties. It is also important to understand which parameters have strong/weak influence on the model output. The uncertainty analysis is performed by using the Monte Carlo simulation method. Morris method of elementary effect is used for input factor screening. To quantify the effect of the input factors on the model output, a variance based method of sensitivity analysis is used.
\end{abstract}

Index Terms-Model uncertainty, ESP lifted oil field, morris method, variance based sensitivity analysis

\section{INTRODUCTION}

ESP lifted oil field is a complex process. There are multiple oil wells working in parallel and are highly interacting. Each oil well consists of a multi-stage centrifugal pump driven by a three phase electric motor. A schematic of an ESP lifted oil field with four oil wells connected to the common production manifold is shown in Fig. 1. ESP is used to provide sufficient lift to raise the oil from the reservoir to the production manifold. The two booster pumps are used to transport the oil from the production manifold through the transportation pipeline to the separator. A dynamic model of this ESP lifted oil field developed by the authors at [2] is used for the uncertainty and sensitivity analysis in this paper. The model for the induction motor is developed using Krause's model and the pumps are modeled using the data provided by the manufacturer. The model representing the dynamics of an oil well is developed using the first principles methods. The oil well parameters are calculated based on the nominal operating conditions of the oil field when the process attains its steady state. The data from a real oil field are not made easily available and hence model validation could not be performed. The nominal operating conditions of the oil field were assumed as per the experts' suggestions received from Statoil Research Center, Porsgrunn, Norway. The details of the development of the model can be found at [2].

Manuscript received April 12, 2014; revised June 28, 2014.

The authors are with the Department of Electrical Engineering, Information Technology and Cybernetics, Telemark University College, Porsgrunn, Norway (e-mail: roshan.sharma@hit.no, bjorn.glemmestad@hit.no ).

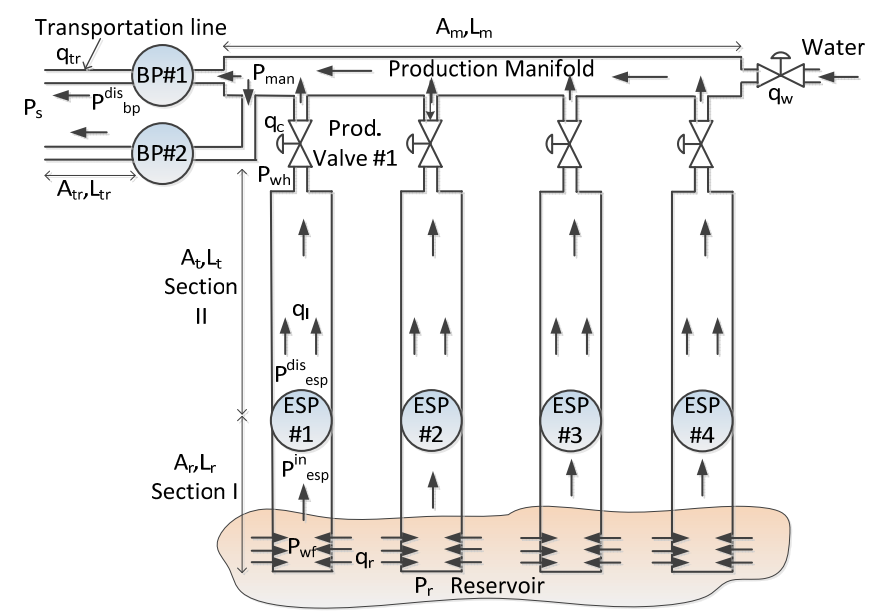

Fig. 1. Schematic of an ESP lifted oil field.

(Courtesy of Statoil Research Center, Porsgrunn, Norway [1])

The model of the oil field is used for understanding the process dynamics and the interactions between the oil wells under varying operating conditions including the cases where certain wells are started up and/or shut down. Furthermore, the model is used for developing optimal control structures for the oil field. Control and optimization of the process is model based. If the mathematical model has uncertain parameters and variables, the results obtained by using the available model may contain uncertainty. For e.g. if the model is used for designing optimal control structure for maximizing total oil production from the oil field by using minimum energy, it becomes interesting to study how uncertain are the optimal values calculated by the model based optimizer and controller if an implementation of the model to a real oil field is considered. At the same time, the modeler or the model user is unsure of how sensitive the output variable is with respect to the changes or variability in the input variables and model parameters. Therefore, it is important to perform uncertainty and sensitivity analysis on the model of the ESP lifted oil field when it is used for estimation, control and optimization. In this paper, we discuss the use of Monte-Carlo simulation method for assessing the model uncertainty. For studying the model sensitivity to input factors, at first Morris' method of elementary effect is used for input factor screening. Later, a variance based sensitivity analysis is performed for quantifying the effect. All the simulations performed for the analysis of the model are based on MATLAB programming language.

The objective of performing uncertainty and sensitivity analysis in the oil field model is to see if the output of the model under consideration lies within a confidence interval when the uncertain parameters of the model are varied. It is 
also to find out which parameters have strong/weak influence on the model output. By understanding the influence of parameters on the output variable, which parameters have to be estimated with more accuracy is known. This can be used for ranking the parameters in order of importance to identify major contributors to the uncertainties in the predictions with a model. Analysis of models for uncertainty and sensitivity is of interest when the model taken into consideration is the result of a long and complex construction process and when the model is used for risk analysis (for example: for calculating the probability of the output from the process to exceed some threshold).

A computational model is required to perform uncertainty and sensitivity analysis. In the appendix, a brief description of the ESP lifted oil field model and the final important equations are given. For a detailed and elaborated development and simulation of the model, refer to [2]. A computational model is a representation of some physical or other system of interest, first expressed mathematically and then implemented in the form of a computer program. In other words, it can be seen as a function of inputs that, when evaluated, produces output [3]. Let the model of the oil field be denoted by a function $f$ with $k$ input factors $x=\left(x_{1}, x_{2}, x_{3}, \ldots, x_{k}\right)$. The total oil produced from the field is considered to be the output $(Y)$ of the model.

$$
Y=f(\mathbf{x})=f\left(x_{1}, x_{2}, x_{3}, \ldots, x_{k}\right)
$$

The behavior of the model is investigated by computational experiments i.e. the output of the model is evaluated by simulating the model (with a computer) at different values of the input factors and some analysis on the simulated model output is performed. The model $f(x)$ is considered to be deterministic. For a given $X$, the model $f(x)$ can be used to evaluate $Y$ without error or in other words, two computations of $Y$ for the same input factors $X$ should be the same [3].

The paper is organized as follows: In Section II, the details of the model uncertainty analysis of the oil field are explained. The various steps used for uncertainty analysis are presented and the simulation results are discussed. The sensitivity analysis is performed using the Morris' method (for input factor screening) and by using the variance based method as described in details in Section III followed by the discussion on the simulation results. Finally, the conclusion of the paper is given in Section IV.

\section{UNCERTAINTY ANALYSIS}

Uncertainty analysis evaluates the uncertainty in the model output for a known or assumed uncertainty in the model components (parameters, input variables) [4]. It can be used to answer the question "What is the uncertainty in the model output given the uncertainty in the input factors?" Input factors for uncertainty analysis are the model parameters or variables whose influence on the model output is to be investigated [4]. The input factors of models are not always known with a sufficient degree of certainty. The uncertainty in the model inputs may be due to variations occurring naturally or due to errors and uncertainties while measuring the input factors. The oil field model consists of four interacting oil wells connected in parallel to the common gathering manifold. For the oil field model, the input factors are:

1) The Productivity Index values of each oil well denoted by $P I_{i}$ where ${ }^{i}$ denotes the $i^{\text {th }}$ oil well.

2) The reservoir pressure $\left(P_{r}\right)$.

3) The water cut $\left(W C_{i}\right)$ values of each oil well.

These parameters and variables directly influence the model output and are thus considered as the input factors. There are a total of nine input factors taken into consideration for uncertainty analysis. The main objective to use the oil field model is to find the optimal working conditions for maximizing the profit obtained from the oil field. The profit from the oil field is directly dependent on the total amount of oil produced from the field. Thus, the model output of high interest for the analysis is the total amount of oil produced from the field. The uncertainty in the input factors should be defined. For the parameters of the oil field, the uncertainty range represents the set of possible values for an input factor. The uncertainty range for each input factor is defined as $\pm 10 \%$ of their respective nominal values. The nominal values of the input factors corresponds to the operating condition when the pump of each well is running at $60 \mathrm{~Hz}$ and the production choke valve of each well is $100 \%$ opened. The values of the input factors for nominal field operation as well their uncertainty ranges are listed in Table I. A continuous probability distribution is defined over each of these uncertainty ranges as described in Section II-A. For the uncertainty analysis the following steps are followed:

1) Define the probability distribution of the input factors.

2) Generate the input scenarios using random sampling.

3) Calculate the total oil production for each input scenario using the oil field model.

4) Analyze the model outputs.

\section{A. Define the Probability Distribution of the Input Factors}

Since the uncertainty range is equal in both the left and right directions from the nominal value, symmetric distribution is well suited for defining the probability distribution of the input factors. The extreme values of the uncertainty ranges are less likely to occur than the middle values. Thus, among the symmetric distribution, Normal or Gaussian distribution is used with specified mean and standard deviation values. A standard Gaussian distribution has a range $[-\infty+\infty]$ but the uncertainty ranges of the input factors are finite. Thus, the standard Gaussian distribution is truncated so that the values of the distribution are within the extreme bounds of the uncertainty ranges. For illustration, the probability distribution of the input factor $P I_{i}$ is shown in

Fig. 2. The mean and the standard deviation values used for creating the distribution of the input factors $P I_{i}$ for each oil well are shown. 


\section{B. Generate the Input Scenarios Using Random Sampling}

Representative samples of the input factors are drawn from their truncated normal distributions by using random sampling. In fact by using MATLAB, the truncated Gaussian distribution for an input factor is at the first place generated by using random number generation. Fig. 2 is then drawn after the randomly distributed normal distributions are sorted in ascending manner. The random samples for the truncated Gaussian distribution are generated independently for each input factor. A total of 10000 input scenarios are generated.

\begin{tabular}{|c|c|c|c|c|c|c|c|c|c|}
\hline $\begin{array}{l}\text { input } \\
\text { factors }\end{array}$ & $\begin{array}{c}P_{r} \\
{[\mathrm{bar}]}\end{array}$ & $P I_{1}$ & $\begin{array}{r}P I_{2} \\
\times\end{array}$ & $\begin{array}{c}\mathrm{PI}_{3} \\
\mathrm{~m}^{5} / \mathrm{Ns}\end{array}$ & $P I_{4}$ & $W C_{1}$ & $W C_{2}$ & $W C_{3}$ & $W C_{4}$ \\
\hline nominal values & 220 & 0.55 & 0.9 & 0.41 & 0.64 & 0.2 & 0.3 & 0.35 & 0.15 \\
\hline $\min$ & 198 & 0.495 & 0.81 & 0.369 & 0.576 & 0.18 & 0.27 & 0.315 & 0.135 \\
\hline $\max$ & 242 & 0.605 & 0.99 & 0.451 & 0.704 & 0.22 & 0.33 & 0.385 & 0.165 \\
\hline
\end{tabular}

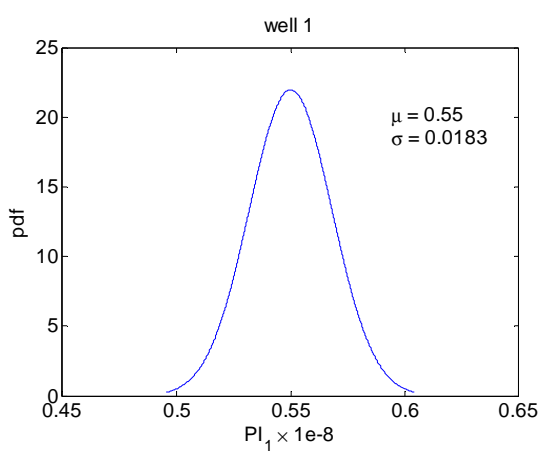

(a) well 1 .

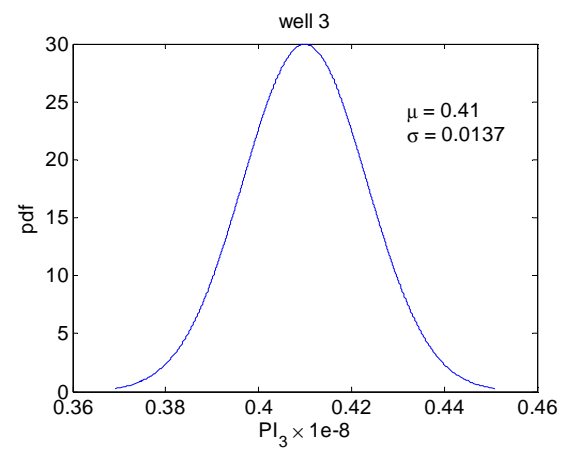

(c) well 3 .

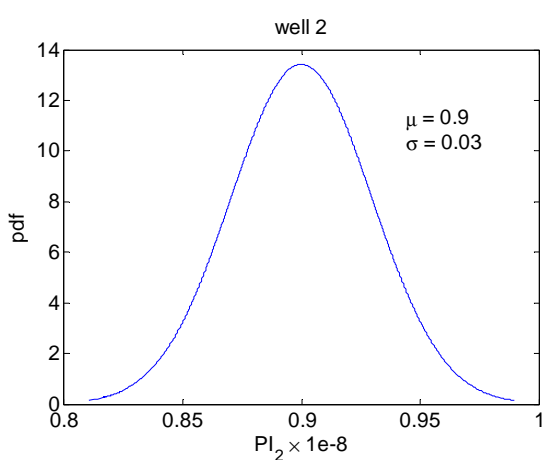

(b) well 2 .

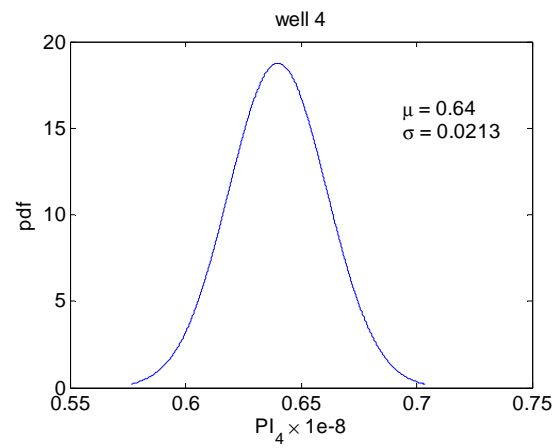

(d) well 4 .

Fig. 2. Probability distribution functions of the input factor $P I_{i}$ for each oil well.

\begin{tabular}{|c|c|c|c|c|c|c|}
\hline $\begin{array}{l}\text { model } \\
\text { output }\end{array}$ & Mean & $\begin{array}{l}\text { Standard } \\
\text { Deviation }\end{array}$ & Min & Max & $1^{\text {st }}$ Quartile & $3^{\text {rd }}$ Quartile \\
\hline$q o_{t o t}$ & 5638.3 & 196.9354 & 4961 & 6197.2 & 5508.4 & 5773.2 \\
\hline
\end{tabular}

\section{Generate the Input Scenarios Using Random Sampling}

Representative samples of the input factors are drawn from their truncated normal distributions by using random sampling. In fact by using MATLAB, the truncated Gaussian distribution for an input factor is at the first place generated by using random number generation. Fig. 2 is then drawn after the randomly distributed normal distributions are sorted in ascending manner. The random samples for the truncated Gaussian distribution are generated independently for each input factor. A total of 10000 input scenarios are generated.

\section{Calculate the Total Oil Production for Each Input Scenario Using the Oil Field Model}

The model of the oil field is simulated 10000 times using one input scenario at a time to obtain 10000 corresponding values of the model output which is the total oil produced from the field $\left(q o_{t o t}\right)$. The model of the oil field is dynamic and consists of ordinary differential equations (ODE) to be solved for each input scenario. The simulation is set up by providing sufficient time for the ODE solvers to ensure that the final value obtained is the steady state value.

\section{E. Analyze the Model Output}

After the simulation is completed for all the input scenarios, the model output can be analyzed by using statistical tools. The average total oil produced from the oil field is $5638.3 \mathrm{Sm}^{3} /$ day and the standard deviation of the output distribution is $196.9354 \mathrm{Sm}^{3} /$ day. Table II shows some statistics on the model output.

The histogram of the output distribution with confidence intervals is shown in Fig. 3. Let us consider the output confidence interval to be in the range $[53005900] \mathrm{Sm}^{3} /$ day. This range can be however defined and adjusted by the 
operator depending on the requirements. The confidence that the total amount of oil produced from the oil field will lie inside this interval is $86.12 \%$. When the ESP of each well is running at $60 \mathrm{~Hz}$ and when the production choke valve of each well is fully opened, there is only a $4.9 \%$ probability that the total oil produced from the oil field will be less than 5300 $\mathrm{Sm}^{3} /$ day for a $\pm 10 \%$ uncertainty in the oil well model input factors. At the same time, the probability that the total oil production will be greater than $5900 \mathrm{Sm}^{3} /$ day is only $8.98 \%$.

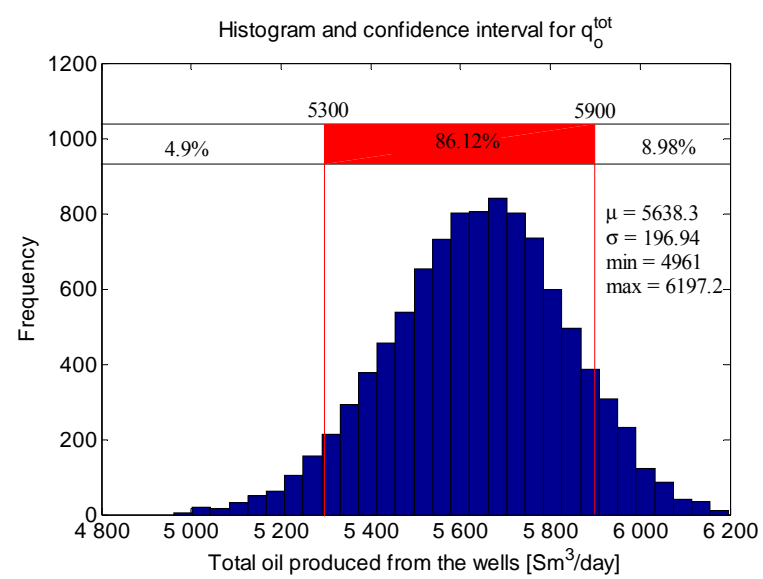

Fig. 3. Histogram plot of the total oil production from the oil field.

In other words, the probability that the output value will deviate from its mean value of $5638.3 \mathrm{Sm}^{3} /$ day by $6 \%$ to its left ( $\approx 5 \%$ to its right) is $86.12 \%$.

It is also interesting to calculate the first and the third quartiles of the output distribution. The first quartile for the total oil production is $5508.4 \mathrm{Sm}^{3} /$ day and the third quartile is $5773.2 \mathrm{Sm}^{3} /$ day. The probability that the output value will lie between the $1^{\text {st }}$ and the $3^{\text {rd }}$ quartile is $50 \%$ i.e. the modeler is sure that half of the output values will be in between 5508.4 $\mathrm{Sm}^{3} /$ day and $5773.2 \mathrm{Sm}^{3} /$ day.

\section{Sensitivity AnAlysis}

In addition to the knowledge about how uncertain the output of a model will be, given an uncertainty in the input, it is also useful to see which parameter will have large/small influence on the model output. Sensitivity analysis deals with evaluating how sensitive are the output of a model with respect to variability in the model input factors. In understanding the behavior of the model, it is useful to discover which inputs of the model are important and have a substantial influence on the outputs [3]. For the case of the oil field model, it is important to identify and screen the important input variables and parameters of the oil field that have significant effect on the total oil production from the field. It is also necessary to quantify the effect of different sources of uncertainty in the oil field model inputs on the variability of the model output.

The input factors that are considered for the uncertainty analysis are also used for the sensitivity analysis. In addition, the speed of the submersible pump $\left(f r_{i}\right)$ and the production choke valve opening of each oil well $\left(u_{i}\right)$ are also taken into consideration. The nominal speed of the pump for each oil well is $60 \mathrm{~Hz}$ and the nominal production choke valve opening is $100 \%$ which is also its maximum opening. For sensitivity analysis, the uncertainty range for $P_{r}$ is taken to be $\pm 10 \%$ from its nominal value. The uncertainty range for the remaining input factors are taken to be $\pm 20 \%$ from their respective nominal values. However, the uncertainty range for the production choke valve opening is taken to be within $-20 \%$ from its nominal value to its maximum opening because the production choke valve cannot be opened by more than $100 \%$. Table III lists the nominal values and their uncertainty ranges for all the inputs factors taken into consideration for the sensitivity analysis.

Local sensitivity analysis is based on the local derivative of the output of $Y$ with respect to $X$. It only gives information about the behavior of $Y$ around given value of $X$. The interest lies on global sensitivity analysis that explores the full input factor space because the purpose of the analysis is to study the effect of uncertainty of several input factors on the model output. In this paper, two widely used methods of global sensitivity analysis are considered. They are: i) The elementary effect method (also known as Morris method) ii) The variance based sensitivity analysis.

\section{A. Morris' Method of Elementary Effect}

Morris' method for computational experiment is used for input factor screening. The input factors are ranked in the order of their importance [3]. But the sensitivity measure using Morris' method is only qualitative and they are not quantified (how much a given factor is more important than others) [5]. It is based on setting up computational experiments that are composed of individually randomized one-factor-at-a-time designs in the input variables and parameters. The data analyses can be based on examination of changes in the output that is unambiguously attributed to changes in the individual inputs so called elementary effects [3]. In other words, Morris' method is the same as calculating one-at-a-time local sensitivity criteria for a lot of different input scenarios. The input scenarios are created by varying the input factors in their whole range of uncertainty domains. Thus Morris method provides global sensitivity analysis.

Each input factor is defined by uniform probability distribution within its uncertainty domain. For calculating the influence of an input factor on the model output, random sampling of the input factors from their uniform probability distribution is performed independently. We have considered 1000 different input scenarios for each input factors.

Let the input factors $\left(P_{r}, P I_{i}, W C_{i}, f r_{i}, u_{i}\right)$ for the oil field model be represented by a vector $X=\left(x_{1}, x_{2}, \ldots, x_{k}\right), i=1,2,3, \ldots, k$. Let the total oil produced from the oil field be represented by $f\left(x_{1}, x_{2}, \ldots, x_{i}, x_{i+1}, \ldots, x_{k}\right)$. The input factor $X$ is scaled to take on values in the interval $[0,1]$ and the sampling domain is a $k$ dimensional $p$ level unit hypercube. According to Morris, the elementary effect of the $i^{\text {th }}$ input factor can be calculated as,

$$
\begin{array}{r}
E E_{i}(X)=\frac{f\left(x_{1}, x_{2}, \ldots, x_{i}+\Delta, x_{i+1}, \ldots, x_{k}\right)}{\Delta} \\
-\frac{f\left(x_{1}, x_{2}, \ldots, x_{i}, x_{i+1}, \ldots, x_{k}\right)}{\Delta}
\end{array}
$$


TABLE III: INPUT FACTORS FOR SENSITIVITY ANALYSIS AND THEIR NOMINAL VALUES AND UNCERTAINTY RANGES

\begin{tabular}{llllll}
\hline $\begin{array}{l}\text { Input } \\
\text { factor }\end{array}$ & Nominal Value & Range & $\begin{array}{l}\text { Input } \\
\text { factor }\end{array}$ & Nominal Value & Range \\
\hline$P_{r}$ & 220 & {$\left[\begin{array}{ll}198 & 242\end{array}\right]$} & $W C_{1}$ & 0.2 & {$\left[\begin{array}{ll}0.16 & 0.24\end{array}\right]$} \\
$P I_{1}$ & $0.55 \mathrm{e}-8$ & {$\left[\begin{array}{ll}0.44 & 0.66\end{array}\right] \mathrm{e}-8$} & $W C_{2}$ & 0.3 & {$\left[\begin{array}{ll}0.24 & 0.36\end{array}\right]$} \\
$P I_{2}$ & $0.9 \mathrm{e}-8$ & {$\left[\begin{array}{ll}0.72 & 1.08\end{array}\right] \mathrm{e}-8$} & $W C_{3}$ & 0.35 & {$\left[\begin{array}{lll}0.28 & 0.42\end{array}\right]$} \\
$P I_{3}$ & $0.41 \mathrm{e}-8$ & {$\left[\begin{array}{lll}0.328 & 0.492\end{array}\right] \mathrm{e}-8$} & $W C_{4}$ & 0.15 & {$\left[\begin{array}{ll}0.12 & 0.18\end{array}\right]$} \\
$P I_{4}$ & $0.64 \mathrm{e}-8$ & {$\left[\begin{array}{lll}0.512 & 0.768\end{array}\right] \mathrm{e}-8$} & $f r_{i}$ & 60 & {$\left[\begin{array}{ll}48 & 72\end{array}\right]$} \\
& & & $u_{i}$ & 100 & {$\left[\begin{array}{ll}80 & 100\end{array}\right]$} \\
\hline \hline
\end{tabular}

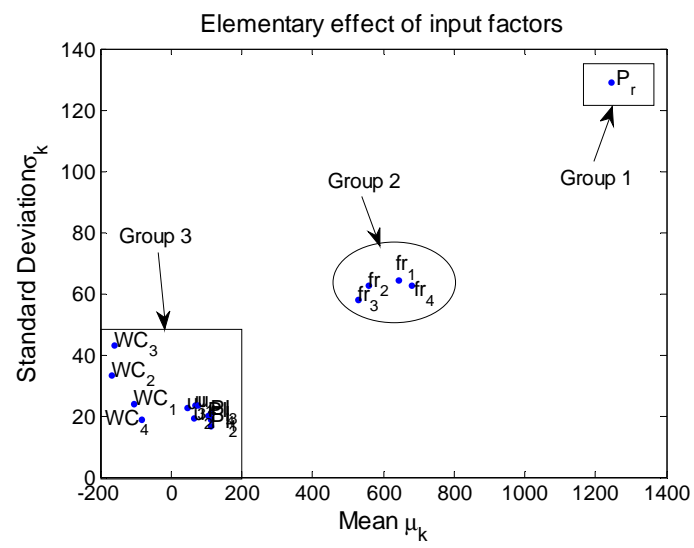

(a) All input factors

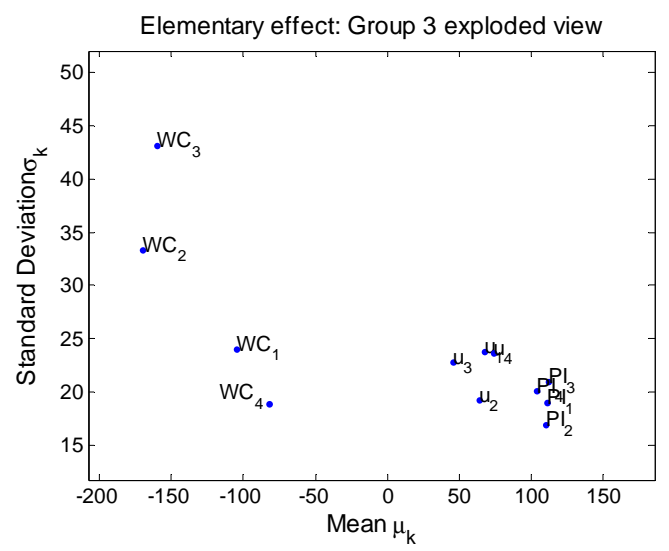

(b) Group 3 exploded view

Fig. 4. Mean and standard deviation of the elementary effect of input factors.

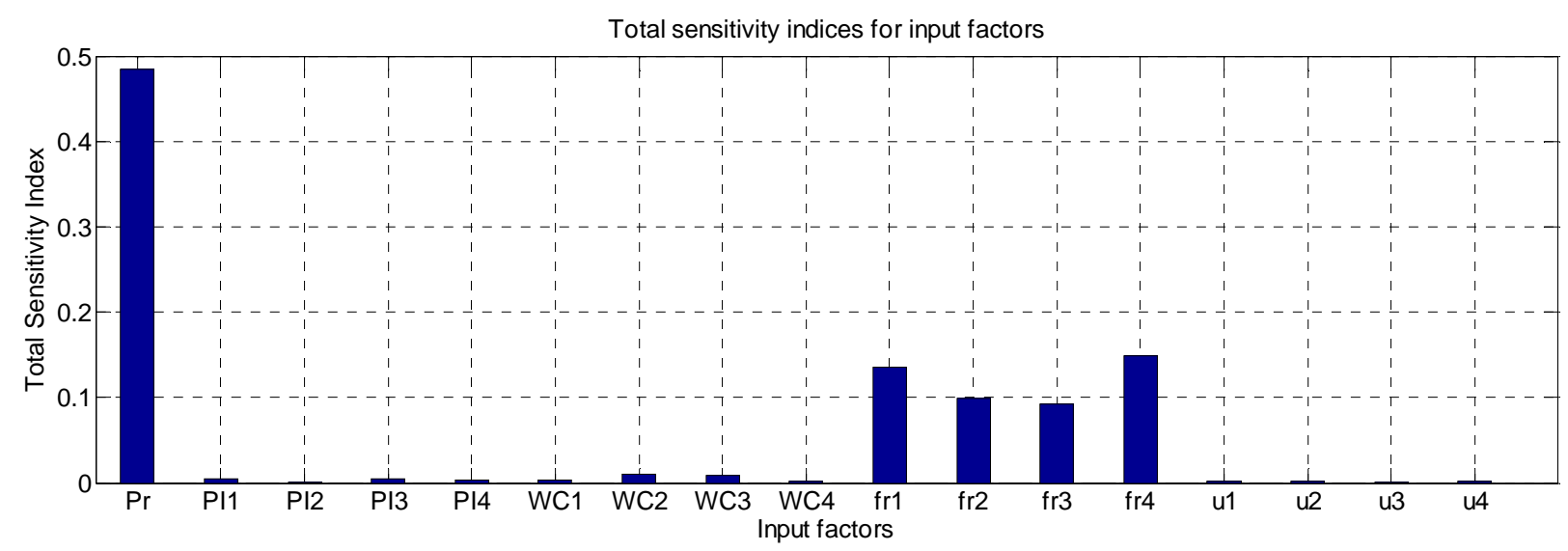

Fig. 5. Total sensitivity index of the input factors of the oil well model.

where $x_{i}+\Delta$ is the perturbed value of $x_{i}$ and $\Delta$ is a predefined multiple of $1 /(p-1)$. Each $x_{i}$ can take a value between 0 and $1-\Delta$. The elementary effect $E E_{i}(X)$ for each $i^{\text {th }}$ input factor is calculated for several randomly selected values of $X$ Morris suggested that the number of runs $r$ is proportional to $k$. A sampling strategy developed by Morris is used to create random samples of $X$ from the input factor sampling domain. Details of the plans for independent random sampling can be found at [3]. To compute $r$ elementary effects of all the $k$ input factors, in the simplest form $2 r k$ model evaluations are needed. $f$ has to be evaluated twice, once at the selected values and once after increasing $x_{i}$ by $\Delta$. However, with Morris' randomized one at a time sampling method, only $r(k+1)$ model evaluations are needed. For the case of oil field model, $p=4, k=17$ and $r=30$ have been used.

The mean and the standard deviation of the $r$ elementary effects of each input factors are calculated. If the mean of the elementary effects is relatively large and the standard deviation is relatively small, the effect of $x_{i}$ on $Y$ is "mildly nonlinear". If the mean is relatively small and the standard deviation is relatively large, then the effect of $x_{i}$ on $Y$ is "strongly nonlinear" [5]. In general, a large measure of the central tendency i.e. mean of the elementary effects indicates an input with an important (overall) influence on the output. A high measure of spread (standard deviation) of $E E_{i}(X)$ may indicate an input factor which is highly interacting with another input factor. It may also indicate that the effect of the input factor is non-linear [3].

The plot of mean and the standard deviation of the elementary effects of each input factor are shown in Fig. 4(a). It can be seen that the input factors of the oil well model can be distinguished into three major groups. Group 1 which is the pressure of the reservoir has the highest mean and the highest standard deviation. This means that it is the most 
influential input factor. A large value of its mean indicates that reservoir pressure has the most important effect on the production of the oil from the field. All the oil wells of the field are assumed to be connected to the same reservoir (with same reservoir pressure for each oil well). Thus any changes or uncertainty in the reservoir pressure is affecting all the wells of the field simultaneously. This is indicated by a large value of its standard deviation which suggests that it is highly interacting with other input factors.

The operating speed of the pumps of each oil wells fall under group 2 which have the second highest mean and the standard deviation values. The effect of the speed of the pumps on the total oil production is lower than that of the reservoir pressure but higher than the remaining input factors They show comparatively more interactions or nonlinear effect than input factors falling under group 3. So the speed of the pumps can have significant influence on the amount of fluid being pumped from the oil wells. Furthermore, from the simulations results of the mathematical model of the oil field as described in [2], it can be seen that if the speed of the pump of an oil well is changed, it not only affects its own pumping rate but also significantly affects all the remaining oil wells of the field (in terms of flow rates and pressures). For an example, an increase in the speed of the pump of one oil well increases the production of oil from that well but at the same time the production of oil from the remaining oil wells are slightly reduced [2]. This interaction may be justified by their relatively higher standard deviation values as can be seen in Fig. 4(a). For the purpose of control and optimization of the operation of the oil field, the speed of the pumps is the input variables that are manipulated or varied.

Finally, the group which has relatively lower means and the standard deviation values is group 3 with consists of water cuts, productivity indices and production choke valve openings as the input factors. The exploded view of the input factors of group 3 is shown in Fig. 4(b). The water cut values have negative mean which shows that its effect on the total oil production is inversely proportional. An increase in the value of water cut of an oil well means a reduction in the production of crude oil from the well as given by the relation,

$$
q_{o}=(1-W C) q_{l}
$$

Here $q_{l}$ is the multiphase fluid being pumped from the oil well. The production choke valves opening of the oil wells and their productivity index values have lower mean and are less sensitive on the production of oil from the field. Compared to the speed of the pumps and the water cut values, they also have lower standard deviation values. So the production choke valves and the productivity index values do not have larger interacting and nonlinear effect on the output from the oil field. In [6]-[8], it has been shown that for production maximization, the choke valves should be always fully opened. During the operation, for most of the time, the production choke valves are fully opened. They are only used when wells have to be shut down or only sometimes used for fluid flow rate control by slightly choking the valve [8]. The Productivity Index (PI) values of the oil wells also have relatively larger mean but almost equal standard deviation when compared to the production choke valve openings.
They affect the production of oil from the field more than the production choke valve opening but less than water cut values. Their lower standard deviation values indicate that they are non-interacting and the effect is not nonlinear.

From Fig. 4, it can be stated that the estimation of the reservoir pressure should be performed with highest importance. The speed of the pumps should also be measured with higher accuracy if it is to be used for the purpose of control and optimization as has been done in [6]-[8].

TABLE IV: SENSITIVITY INDICES FOR THE INPUT FACTORS OF THE OIL WELL

\begin{tabular}{cccccc}
\multicolumn{6}{c}{ MODEL } \\
\hline $\begin{array}{c}\text { input } \\
\text { factor }\end{array}$ & $S_{i}$ & $S_{T i}$ & $\begin{array}{c}\text { input } \\
\text { factor }\end{array}$ & $S_{i}$ & $S_{T i}$ \\
\hline$P_{r}$ & 0.4839 & 0.4864 & $f r_{1}$ & 0.1352 & 0.1358 \\
$P I_{1}$ & 0.0035 & 0.0042 & $f r_{2}$ & 0.0997 & 0.1000 \\
$P I_{2}$ & 0.0008 & 0.0012 & $f r_{3}$ & 0.0912 & 0.0923 \\
$P I_{3}$ & 0.0037 & 0.0043 & $f r_{4}$ & 0.1467 & 0.1492 \\
$P I_{4}$ & 0.0030 & 0.0036 & $u_{1}$ & 0.0001 & 0.0016 \\
$W C_{1}$ & 0.0012 & 0.0038 & $u_{2}$ & 0.0010 & 0.0014 \\
$W C_{2}$ & 0.0086 & 0.0096 & $u_{3}$ & 0.0007 & 0.0008 \\
$W C_{3}$ & 0.0072 & 0.0090 & $u_{4}$ & 0.0011 & 0.0020 \\
$W C_{4}$ & 0.0016 & 0.0023 & & & \\
\hline \hline
\end{tabular}

\section{B. Variance Based Method of Sensitivity Analysis}

The Morris' method described in Section III-A only qualitatively identifies the influence of the model input factors on the output. To quantify the effect of the input factors on the model output, variance based global sensitivity analysis methods can be used. Let us consider the model $Y=f\left(x_{1}, x_{2}, \ldots, x_{k}\right)$ where $x_{1}$ to $x_{k}$ be $k$ independent input factors. The input factor space is assumed to be a $k$-dimensional unit hypercube. The method is based on full decomposition of the variance of $Y$ into terms depending on the factors and their interactions as detailed in [9].

$$
V(Y)=\sum_{i} V_{i}+\sum_{i} \sum_{j>i} V_{i j}+\ldots+V_{12 \ldots k}
$$

Here, $V(Y)$ is the unconditional variance of the output and is written as [10],

$$
V(Y)=V_{X_{i}}\left(E_{X_{\sim i}}\left(Y \mid X_{i}\right)\right)+E_{X_{i}}\left(V_{X_{\sim i}}\left(Y \mid X_{i}\right)\right)=V(Y)
$$

Comparing (4) and (5), $V_{i}=V_{X_{i}}\left(E_{X_{\sim i}}\left(Y \mid X_{i}\right)\right)$ measures the first order or main effect of $X_{i}$ on the model output. $X_{\sim i}$ denotes all the factors but $X_{i}$. The second term of (5) is called the residual. $E_{X_{\sim i}}\left(Y \mid X_{i}\right)$ indicates that the mean of $Y$ is taken over all possible values of $X_{\sim i}$ by keeping $X_{i}$ fixed and $V_{X_{i}}\left(E_{X_{\sim i}}\left(Y \mid X_{i}\right)\right)$ is the expected reduction in variance if $X_{i}$ is fixed. By normalizing the first order effect by $V(Y)$, the first order sensitivity coefficient $\left(S_{i}\right)$ is written as [11],

$$
S_{i}=\frac{V_{X_{i}}\left(E_{X_{\sim i}}\left(Y \mid X_{i}\right)\right)}{V(Y)}
$$

The first order effect measures the additive effect of $X_{i}$ 
on the model output. The interactions between the input factors are not taken into consideration. The sum of the first order indices of a linear model is one. The oil field model is nonlinear and it is of interest to take into account the interactions and the total effect of input factors on the model output. We are focused on measuring the total sensitivity index which includes the first and the higher order effects of factor $X_{i}$. It can be calculated as [12],

$$
S_{T i}=\frac{E_{X_{\sim i}}\left(V_{X_{i}}\left(Y \mid X_{i}\right)\right)}{V(Y)}
$$

The term on the numerator of (7) is the expected variance that would be left if all the factors could be fixed except $X_{i}$. There are numerous literature on the formulation of the estimators to estimate $V_{X_{i}}\left(E_{X_{\sim i}}\left(Y \mid X_{i}\right)\right)$ and $E_{X_{\sim i}}\left(V_{X_{i}}\left(Y \mid X_{i}\right)\right)$ for the calculation of $S_{i}$ and $S_{T i}$ respectively as can be found in [9], [11], [13]-[15]. In this paper, the estimators formulated in [11] are used. The $V(Y)$ and $S_{T i}$ are computed or approximated with Monte Carlo simulations.

Let $r$ be the number of simulations and $k$ the number of input factors. We consider two independent sampling matrices $A$ and $B$ each of size $(r, k)$. In order to generate samples of $X_{1}, X_{2}, \ldots, X_{k}$ as uniformly as possible over the unit hypercube, Sobols' quasi-random sequences are used [16]. Matrices $A$ and $B$ can be generated from Sobols' quasi random sequence of size $(r, 2 k)$ such that $A$ is the left half of the sequence and $B$ is the right half of it. $k$ Number of third matrices $A_{B}^{(i)}, i=1,2, \ldots, k$ are created where all columns are from $A$ except the $i^{\text {th }}$ column which is from matrix $B$. We then have the triplet $A, B$ and $A_{B}^{(i)}$ that will be used to calculate the sensitivity indices. At first, the $V(Y)$ can be calculated as,

$$
\hat{f}_{0}=\frac{1}{N} \sum_{j=1}^{N} f(A)_{j}, \quad \hat{V}(Y)=\frac{1}{N} \sum_{j=1}^{N}\left(f(A)_{j}\right)^{2}-\hat{f}_{0}^{2}
$$

$$
\text { The estimates of } V_{X_{i}}\left(E_{X_{\sim i}}\left(Y \mid X_{i}\right)\right) \quad \text { and }
$$

$E_{X_{\sim i}}\left(V_{X_{i}}\left(Y \mid X_{i}\right)\right)$ for the calculation of $S_{i}$ and $S_{T i}$ according to [11] are respectively,

$$
\begin{gathered}
V_{X_{i}}\left(E_{X_{\sim i}}\left(Y \mid X_{i}\right)\right) \approx \frac{1}{N} \sum_{j=1}^{N} f(B)_{j}\left(f\left(A_{B}^{(i)}\right)_{j}-f(A)_{j}\right) \\
E_{X_{\sim i}}\left(V_{X_{i}}\left(Y \mid X_{i}\right)\right) \approx \frac{1}{2 N} \sum_{j=1}^{N}\left(f(A)_{j}-f\left(A_{B}^{(i)}\right)_{j}\right)^{2}
\end{gathered}
$$

The total sensitivity index can be found using (7) and (10). To compute the model output $Y$ for matrices $A$ and $B, 2 r$ simulations are required. To calculate $Y$ for $A_{B}^{(i)}$ for all the input factors, $r k$ simulations are needed. The total number of model computation becomes $r(k+2)$. for the case of oil field model with $r=10000$ and $k=17$ (input factors), a total of 190,000 model computations are needed. Table IV shows the first order and the total sensitivity indices of all the 17 input factors of the oil field. The total sensitivity indices have slightly higher value than their corresponding first order sensitivity indices for all the input factors. For easier representation and comparison, the bar diagram of the total sensitivity indices is shown in Fig. 5.

The total sensitivity indices are clearly separated into three categories by the heights of the vertical bars (like in the case with Morris elementary method). The total sensitivity index for the reservoir pressure is 0.4864 and is the largest of all the input factors. The total sensitivity indices for the speed of the pumps are the second largest ranging from 0.0923 to 0.1492 . The remaining input factors have relatively smaller values of the total sensitivity indices. The production of the oil from the field is most sensitive to the reservoir pressure and least sensitive to the production choke valve openings.

\section{CONCLUSION}

In this paper, the study of the uncertainty and the sensitivity analysis of the model of an ESP lifted oil field are performed using Monte Carlo simulation methods. From the uncertainty analysis, it was seen that a confidence interval of $86.12 \%$ can be achieved with a $\pm 10 \%$ uncertainty in the input factors. Among the input factors, the reservoir pressure is the most sensitive and important factor. It should be estimated with highest accuracy. The speed of the pumps also has a significant influence on the total oil production from the field. The speed of the pumps of each oil well should also be measured with sufficient accuracy. Two different methods of sensitivity analysis showed the same results for the model of the oil field. Morris screening method based on the calculation of elementary effect requires far less model computations than the variance based method. For the model of the oil field taken into consideration, it is sufficient to use Morris method for sensitivity analysis.

\section{APPENDIX}

Mathematical Model Of The Esp Lifted Oil Field.

The ESP lifted oil field consists of modeling the electric submersible pump, electric motor, booster pump and different sections of the oil well including the riser pipe, gathering manifold and transportation lines. Detailed modeling and simulation of the oil field can be found in another article written by the authors at [2]. In this paper, only the final equations of the model of the oil field have been rewritten as also explained in the papers by the author at [6] and [17]. The superscript $i$ and $j$ denote the $i^{\text {th }}$ oil well and the $j^{\text {th }}$ transportation pipeline.

$$
\frac{d(2 \pi f)}{d t}=\frac{P}{2 J}\left(T_{e}-T_{l}\right)
$$

\section{A. Motor Model}

The rotor shaft of the electric motor is coupled with the ESP. The rotation of the motor shaft will provide the rotational speed $(f)$ to the ESP. For a given oil well, the speed of the pump can be written as, 
Here, $P=2$ is the number of poles of the induction motor, $J$ is the moment of inertia of the rotor shaft and $T_{l}$ is the load torque. The electric torque $\left(T_{e}\right)$ is given by,

$$
T_{e}=\frac{3}{2} \frac{P}{2} \frac{1}{w_{b}}\left(\psi_{d s}^{e} i_{q s}^{e}-\psi_{q s}^{e} i_{d s}^{e}\right)
$$

Here, $w_{b}=60 \mathrm{~Hz}$ is the base speed. Krause's model [18] is used to calculate the d-axis flux linkage in stator $\left(\psi_{d s}^{e}\right)$, q-axis flux linkage in stator $\left(\psi_{q s}^{e}\right)$, q-axis current in stator $\left(i_{q s}^{e}\right)$ and d-axis current in stator $\left(i_{d s}^{e}\right)$.

\section{B. ESP Model}

Pump 538-11000 provided by Premier Pumping Solutions [19] is used for each oil well. The manufacturer provides data only for a single stage of ESP when it is pumping water. Hydraulic Institute Model [20] for viscosity correction is used to correct the pump characteristics for pumping a viscous fluid from the reservoir. For a multi-stage ESP, the head characteristics $\left(H_{e s p}^{i}(Q, f)\right)$ of the $i^{\text {th }}$ ESP after the viscosity correction at any speed $f$ is written as a third order polynomial as,

$$
H_{e s p}^{i}(Q, f)=\frac{\bar{a}_{0}^{i}}{f_{0}^{2}} f^{2}+\frac{\bar{a}_{1}^{i}}{f_{0}} f Q(f)+\bar{a}_{2}^{i} Q^{2}(f)+\frac{\bar{a}_{3}^{i}}{f} f_{0} Q^{3}(f)
$$

Here, $\bar{a}_{0}^{i}, \bar{a}_{1}^{i}, \ldots, \bar{a}_{3}^{i}$ are the polynomial coefficients for the base frequency $f_{0}=60 \mathrm{~Hz}$ and $Q(f)$ is the fluid flow rate through the ESP. The Brake Horse Power (BHP) characteristic $\left(B H P_{e s p}^{i}(Q, f)\right)$ of the $i^{\text {th }}$ ESP after the viscosity correction at any given frequency $f$ written as a fourth order polynomial,

$$
\begin{array}{r}
B H P_{e s p}^{i}(Q, f)=\frac{\hat{a}_{0}^{i}}{f_{0}^{3}} f^{3}+ \\
+\frac{\hat{a}_{1}^{i}}{f_{0}^{2}} f^{2} Q(f)+\frac{\hat{a}_{2}^{i}}{f_{0}} f Q^{2}(f) \\
+\hat{a}_{3}^{i} Q^{3}(f)+\frac{\hat{a}_{4}^{i}}{f} f_{0} Q^{4}(f)
\end{array}
$$

Here, $\hat{a}_{0}^{i}, \hat{a}_{1}^{i}, \ldots, \hat{a}_{4}^{i}$ are the polynomial coefficients for the base frequency $f_{0}=60 \mathrm{~Hz}$. The minimum $\left(Q_{\min }^{i}(f)\right)$ and the maximum $\left(Q_{\max }^{i}(f)\right)$ viscous fluid flow rate through the ESP for any given frequency $f$ can be calculated as,

$$
Q_{\min }^{i}(f)=\frac{f}{f_{0}} Q_{f_{0}, \min }^{i} \quad Q_{\max }^{i}(f)=\frac{f}{f_{0}} Q_{f_{0}, \max }^{i}
$$

Here, $Q_{f_{0} \text {, min }}^{i}$ and $Q_{f_{0} \text {, max }}^{i}$ are the minimum and maximum flow rates through ESP pumping viscous fluid at $f_{0}$. The head generated and the BHP consumed by the pump for different speeds can be calculated using the Affinity laws [21], [22].

\section{Well Model}

Four oil wells each connected to the common production manifold are considered. The differential equations representing the dynamics of each $i^{\text {th }}$ oil wells are,

$$
\begin{gathered}
\dot{q}_{l}^{i}=\frac{A_{t}^{i}}{\rho_{l}^{i}\left(L_{r}^{i}+L_{t}^{i}\right)}\left\{P_{w f}^{i}-P_{w h}^{i}+\rho_{l}^{i} g H_{e s p}^{i}\left(q_{l}^{i}, f_{r}^{i}\right)-\rho_{l}^{i} g L_{r}^{i}\right. \\
\left.-\rho_{l}^{i} g L_{t}^{i}-\Delta P_{f}^{r, i}-\Delta P_{f}^{t, i}\right\} \\
\dot{P}_{w f}^{i}=\frac{\beta}{A_{r}^{i} L_{r}^{i}}\left[q_{r}^{i}-q_{l}^{i}\right] \\
\dot{P}_{w h}^{i}=\frac{\beta}{A_{t}^{i} L_{t}^{i}}\left[q_{l}^{i}-q_{c}^{i}\right] \\
\dot{q}_{t r}^{j}=\frac{A_{t r}^{j}}{\rho_{t r}^{j} L_{t r}^{j}}\left[P_{\text {man }}-P_{s}+\rho_{t r}^{j} g H_{b p}^{j}\left(q_{t r}^{j}, f_{b p}^{j}\right)-\Delta P_{f}^{t r, j}\right] \\
\dot{P}_{\text {man }}=\frac{\beta}{A_{m} L_{m}}\left[q_{m a n}^{i n}-\sum_{j=1}^{N_{b p}} q_{t r}^{j}\right]
\end{gathered}
$$

Here, $q_{l}, q_{r}, q_{c}, q_{t r}$ are the average fluid flow rates through a well, from the reservoir into the tubing, through the production choke valve and through the transportation pipeline respectively. $A_{t}, L_{t}, A_{r}, L_{r}$ are the cross sectional areas and lengths of tubing in section I and II (see Fig. 1) respectively. $A_{t r}, L_{t r}, A_{m}, L_{m}$ are the cross sectional areas and lengths of the transportation pipeline and the gathering manifold respectively. $P_{w f}, P_{w h}, P_{\text {man }}, P_{s}$ are the bottom hole, well head, gathering manifold and separator pressures. $H_{e s p}, H_{b p}$ are the head produced by the ESP and the booster pumps. $\rho_{l}, \rho_{t r}$ are the densities of fluid flowing through the well and the transportation line. $\Delta P_{f}^{r}, \Delta P_{f}^{t}, \Delta P_{f}^{t r}$ are the pressure losses due to friction in Section I and II of the tubing and in the transportation pipeline. $f_{r}^{i}$ is the speed of the pump, $\beta$ is the bulk modulus of the reservoir fluid and $q_{\text {man }}^{i n}$ is the total fluid (including the injected water) flowing into the gathering manifold.

$q_{r}^{i}$ can be expressed using the Productivity Index (PI) model [22] and reservoir pressure $P_{r}$ as,

$$
q_{r}^{i}=P I^{i}\left(P_{r}-P_{w f}^{i}\right)
$$

$q_{c}$ can be expressed using the standard flow equation ANSI/ISA S75.01 developed by Instrument Society of America [23] as,

$$
\begin{gathered}
q_{c}^{i}=\bar{N}_{6} C_{v}\left(u^{i}\right) \sqrt{\frac{\max \left(P_{w h}^{i}-P_{\text {man }}, 0\right)}{\rho_{l}^{i}}} \\
C_{v}\left(u^{i}\right)=\left\{\begin{array}{cc}
0 & u^{i} \leq 5 \\
0.111 u^{i}-0.556 & 5<u^{i} \leq 50 \\
0.5 u^{i}-20 & u^{i}>50
\end{array}\right.
\end{gathered}
$$

Here, $\bar{N}_{6}=N_{6} /\left(3600 \sqrt{10^{5}}\right)$ with $N_{6}=27.3$. The valve characteristics as a function of its opening $\left(C_{v}\left(u^{i}\right)\right)$ is modeled by three linear equations by fitting the data supplied 
by the choke supplier as,

The pressure loss due to friction is calculated using Darcy-Weisbach formula [24] as,

$$
\Delta P_{f}=\frac{f_{D} L \rho v^{2}}{2 D_{h}}
$$

Here, $\rho$ is the density of fluid flowing through the pipeline, $D_{h}$ is the hydraulic diameter of the pipe and $v$ is the velocity of the fluid. The Darcy friction factor $f_{D}$ can be evaluated using Serghide's explicit approximation to Coolebrook-white equation [25].

\section{REFERENCES}

[1] A. Pavlov and V. Alstad, "Modelling, simulation and automatic control of ESP lifted wells," Statoil Research Center, Tech. Rep., 2010, Internal Report.

[2] R. Sharma and B. Glemmestad, "Modeling and simulation of electric submersible pump lifted oil field," International Journal of Petroleum Science and Technology, vol. 8, no. 1, pp. 39-68, 2014.

[3] M. D. Morris, "Factorial sampling plans for preliminary computational experiments," Technometrics, vol. 33, no. 2, pp. 161-174, May 1991.

[4] H. Monod, C. Naud, and D. Makowski, Working with dynamic crop models: Evaluation, Analysis, Parameterization and Applications, Eds. Elsevier, 2006

[5] P. A. Ekström, "Eikos: A simulation toolbox for sensitivity analysis in MATLAB," FACILIA AB, Tech. Rep., June 2005.

[6] R. Sharma and B. Glemmestad, "Optimal control strategies with nonlinear optimization for an electric submersible pump lifted oil field," Modeling, Identification and Control, vol. 34, no. 2, pp. 55-67, 2013.

[7] A. A. Linninger, "Nonlinear model predictive control for optimal operation of electric submersible pump lifted oil field," in Proc. the 33rd IASTED International Conference on Modeling, Identification and Control, Innsbruck, Austria, February 17-19, 2014, pp. 229-236.

[8] V. S. Suicmez, "Mixed integer nonlinear programming for ESP lifted oil field and improved operation through valve choking," Journal of Petroleum Science and Engineering, March 2014.

[9] I. M. Sobol', "Sensitivity analysis for non-linear mathematical models," Mathematical modeling and Computational Experiment, vol. 1, pp. 407-414, 1993.

[10] A. M. Mood, F. A. Graybill, and D. C. Boes, Introduction to the Theory of Statistics, $3^{\text {rd }}$ ed. McGraw-Hill, 1974.

[11] A. Saltelli, P. Annoni, I. Azzini, F. Campolongo, M. Ratto and S. Tarantola, "Variance based sensitivity analysis of model output. Design and estimator for the total sensitivity index," Computer Physics Communications, vol. 181, pp. 259-270, 2010.

[12] A. Saltelli and S. Tarantola, "On the relative importance of input factors in mathematical models: Safety assessment for nuclear waste disposal," American Statistical Association, vol. 97, pp. 702-709, 2002.

[13] M. J. W. Jansen, "Analysis of variance design for model output," Computer Physics Communication, vol. 117, pp. 35-43, 1999.

[14] T. Homma and A. Saltelli, "Importance measures in global sensitivity analysis of model output," Reliability Engineering and System Safety, vol. 52 , no. 1, pp. 1-17, 1996.

[15] I. M. Sobol', "Global sensitivity analysis indices for the investigation of nonlinear mathematical models," Matematicheskoe Modelirovanie, vol. 19, no. 11, pp. 23-24, 2007, (in Russian).
[16] S. Kucherenko and Y. Sytsko, "On the distribution of points in a cube and the approximate evaluation of integrals," USSR Computational Mathematics and Mathematical Physics, vol. 7, pp. 86-112, 1967.

[17] R. Sharma and B. Glemmestad, "Nonlinear optimization and control of an electric submersible pump lifted oil field," in Proc. the 5th International Conference on Modeling, Identification and Control, Cairo, Egypt, Aug. 31 - Sep. 2, 2013, pp. 26-31.

[18] P. C. Krause, Analysis of Electric Machinery, McGraw-Hill Book Company, 1986.

[19] Premier Pumping Solutions. (2012). Electric Submersible pump, section 1, pump catalog. [Online]. Available: $\mathrm{http}: / /$ www.premierpumpingsolutions.com/electric_submersible_pum p.html.

[20] Hydraulic Institute, ANSI-HI pump standards, 2012.

[21] I. Karassik and T. McGuire, Centrifufal Pumps, New York, USA: Chapman and Hall, 1998.

[22] G. Takacs, Electric Submersible pump manual: design, operation and maintenance, Jordan Hill, Oxford, UK: Gulf Professional Publishing, 2009.

[23] ANSI/ISA S75.01, Flow Equations for sizing control valves, Standards and Recommended Practices for Instrumentation and Control, $10^{\text {th }}$ ed., 1989, vol. 2.

[24] K. E. Brown and H. D. Beggs, The technology of artificial lift methods, Inflow Performance, Multiphase flow in pipes, the flowing well. Tulsa, Oklahoma, ISBN: 0-87814-031-X: PennWell Publishing Company, 1977, vol. 1

[25] T. K. Serghide, "Estimate friction factor accurately," Chemical Engineering, vol. 91, no. 5, pp. 63-64, 1984.

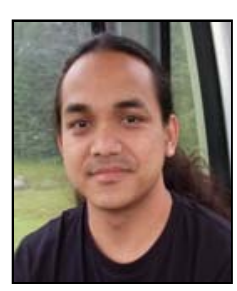

Roshan Sharma was born in 1982 in Nepal. He received his bachelor in engineering from Tribhuvan University in 2007. After about 2 years of academic experience working as an assistant lecturer, he received M.Sc in systems and control engineering from Telemark University College, Norway. Currently he is a PhD student at Telemark University College. His main areas of research are within the field of process modeling, simulation, control and optimization. At the present, he is working on the design and implementation of optimal control structures for artificially lifted oil wells. He is focused on optimal control of Gas lifted and ESP lifted oil fields.

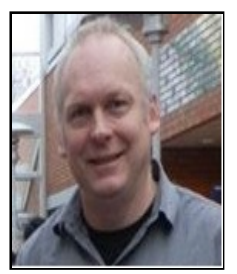

Bjørn Glemmestad was born in Norway. $\mathrm{He}$ received his B.Sc. from Telemark University College in 1986. After six years industrial experience he received a M.Sc. within process automation at Telemark University College in 1994 and his $\mathrm{PhD}$ within process technology from Norwegian University of Science and Technology in 1997. Since then Glemmestad has worked several years within the petrochemical industry being involved in energy and environmental issues in Norsk Hydro AS, but the main focus has been on systems for advanced process control (APC) and process optimization at the Borealis Petrochemical Group. He has been involved in developing proprietary APC technology and implementing this at petrochemical plants in several countries, and he was a manager for the APC Department in the Borealis Group for four years. In 2009 he started as a professor in industrial optimization at Telemark University College and was the head of Department for Process, Energy and Environmental Technology until 2013. Currently, he works at Yara International AS, Porsgrunn, Norway. 